\section{Use of Human Embryonic Stem Cells in the Treatment of Thrombocytopenia: a Case Series}

Geeta Shroff ${ }^{1}$

1 Director, Nutech Mediworld, New Delhi, India.

\section{Contact information:}

Geeta Shroff.

Address: Nutech Mediworld, H-8, Green Park Extension, New Delhi-110016, India. Tel: +91 1126180039 .

Fax: +911126560089.

”geetashroff@hotmail.com

Keywords

Thrombocytopenia; Platelets; Human Embryonic Stem Cells; Immunogenicity.

Thrombocytopenia is defined as the reduction in blood platelet count below the normal platelet count distribution $\left(1.5 \mathrm{~m}^{3}\right)$ [1]. It is the second most common hematological disorder after anemia and equally affects both men and women [2]. The platelets play an important role in blood clotting mechanism. The decrease in the platelet number increases the bleeding and blood loss; and when coupled with other clotting disorders can lead to serious morbidity or death [3]. 
The main causes of thrombocytopenia include ineffective platelet production by bone marrow, increased destruction of platelets and abnormal distribution of platelets. The abnormal distribution or aggregation of platelets can be seen as a result of splenic diseases such as neoplastic, congestive, infiltrative and infections; hypothermia; and massive transfusions [4]. For the treatment of thrombocytopenia, it is important to understand its auto pathology. The treatment of thrombocytopenia patients depends on the causes and severity of the disease. Different treatments such as corticosteroids, immunoglobulins, blood or platelet transfusions, or splenectomy are available for thrombocytopenia. Corticosteroids, mostly prednisone used as the first line of therapy for thrombocytopenia increases the platelet count by decreasing the activity of the immune system $[2,5]$. The main focus of these treatments is to prevent death and disability due to bleeding. However, the use of corticosteroids leads to various adverse events (AEs) such as moon face, bloating, swelling, weight gain, hair loss, depression, hot flushes, visual problem etc [6, 7]. The search for safer, targeted and specific stimulation of thrombopoiesis has gained the attention of researchers and clinicians towards the molecular regulation of different stem cells [8]. Several studies have reported the use of hematopoietic stem cells for the treatment of thrombocytopenia $[9,10]$. Here, in the present study, we report the safety and efficacy of human embryonic stem cell (hESC) therapy in two patients with thrombocytopenia.

\section{Methods}

hESCs used in the present study were obtained from a single, spare, expendable, pre-implantation stage fertilized ovum taken during natural in vitro fertilization (IVF) process after getting an informed consent. The cell lines were cultured and maintained as per our patented in-house technology (United States Granted Patent No US 8592, 208, 52) in a
Good Laboratory Practice (GLP), Good Manufacturing Practice (GMP) and Good Tissue Practice (GTP) compliant laboratory. The derivation and characterization of hESCs has been presented previously [11]. The safety and efficacy of these hESCs has been established in various terminal conditions including cerebral palsy (CP), spinal cord injury (SCI) and nonhealing wounds [12]. The study was approved by independent Institutional Ethics Committee (IEC). A written and video informed consent was obtained from the patients prior to the start of the treatment.

The treatment plan consists of one treatment session with hESC therapy, following an already established treatment protocol [12]. The patients were injected with $1 \mathrm{~mL}$ hESCs daily via an intravenous route (i.v) to help cells reach the injury site (homing area) for repair and regeneration. Bone marrow aspiration and analysis were not performed for these patients, as the underlying condition was serious. Patients were monitored regularly for the occurrence of any antigenic or anaphylactic reaction by the in-house physicians and nurses during the whole therapy.

\section{Case 1}

A 26-year-old male was admitted to our facility in August 2015 with complaints of being unable to lift both arms and forearm above shoulder level, difficulty in getting up from sitting position, weakness of the facial muscles, loss of the muscle mass and winging of the scapula. The patient's clinical history revealed that in June 2012, he was diagnosed with Fascio scapulo humoral dystrophy (FSHD).

The laboratory examination at our facility showed his platelet count as $0.70 \mathrm{~m}^{3}$, that confirmed thrombocytopenia. At our facility, the patient underwent hESC therapy. After the treatment, the patient showed a remarkable improvement in all the symptoms. The patient was able to lift both arms and forearms above the shoulder and could get up at his own. The patient also showed a decrease in the facial muscle weakness, loss of the muscle mass and 
winging of the scapula. The platelet count noted in August 2016 was $1.01 \mathrm{~m}^{3}$ following the treatment. The total blood count before and after the therapy are presented in Table 1. A remarkable improvement was observed in the patient while undergoing hESC therapy, which is difficult to achieve with the currently available therapies.

Table 1. Blood Platelet Count of the Patient Before, During and at the End of Human Embryonic Stem Cell Therapy

\begin{tabular}{|l|c|}
\hline \multicolumn{1}{|c|}{ Date } & PC $\left(\mathbf{m}^{3}\right)$ \\
\hline Case 1 & \\
\hline $14 / 8 / 15$ & 0.70 \\
\hline $18 / 9 / 15$ & 0.94 \\
\hline $24 / 9 / 15$ & 0.84 \\
\hline $30 / 09 / 15$ & 0.93 \\
\hline $1 / 10 / 15$ & 0.86 \\
\hline $5 / 10 / 15$ & 0.88 \\
\hline $8 / 10 / 15$ & 0.98 \\
\hline $22 / 10 / 15$ & 1.31 \\
\hline $20 / 11 / 15$ & 1.55 \\
\hline $2 / 12 / 15$ & 1.26 \\
\hline $8 / 1 / 16$ & 1.38 \\
\hline $10 / 1 / 16$ & 1.59 \\
\hline $3 / 8 / 16$ & 1.01 \\
\hline Case 2 & \\
\hline $19 / 8 / 15$ & 0.8 \\
\hline 9/9/15 & - \\
\hline $11 / 10 / 15$ & - \\
\hline $23 / 10 / 15$ & - \\
\hline $31 / 10 / 15$ & 0.73 \\
\hline $4 / 11 / 15$ & 1.59 \\
\hline $17 / 11 / 15$ & 0.93 \\
\hline $1 / 12 / 15$ & 1.12 \\
\hline $26 / 12 / 15$ & 1.5 \\
\hline $24 / 1 / 16$ & 1.5 \\
\hline $14 / 3 / 16$ & 1.05 \\
\hline $26 / 6 / 16$ & Platelet count. \\
\hline & \\
\hline
\end{tabular}

\section{Discussion}

Thrombocytopenia is a hematological disorder associated with increased destruction, consumption, or decreased platelets production [2]. Currently available treatment options such as corticosteroids, blood or platelet transfusions and immunoglobulins medicines are associated with complications $[6,7]$. Recently, the use of stem cell therapy has enhanced the clinical treatment of human patients with thrombocytopenia [13]. Stem cells are being explored by scientists in regenerative medicine for treating various diseases due to their potential to multiply, proliferate and differentiate into any cell type [14, 15]. At the injured site, stem cells produce different trophic factors and reduce the cell loss, promote host regeneration, hence, 
restore the function [16]. Lim et al., (1997) described complete remission after autologous stem cell transplantation (ASCT) in patients with refractory idiopathic thrombocytopenia (ITP) [9]. Al-Ahmari et al., (2004) transferred allogenic stem cells into patients with congenital amegakaryocytic thrombocytopenia (CAT). It was observed that allogenic stem cells are safe and effective for the treatment of patients with CAT [17].

The proliferation and differentiating ability of hESCs has made this therapy an attractive therapeutic option. Takayama et al., (2008) reported that hESCs efficiently develop into hematopoietic progenitors and megakaryocytes capable of releasing functional platelets [18].

The present study reported the use of in-house cultured hESC therapy in the treatment of two patients with thrombocytopenia. After hESC therapy at our facility, these patients showed a significant increase in blood platelet count which further helps in improvement of symptoms such as breathing, lethargy, fatigue and ability to do routine work. No AEs were reported. The bone marrow aspiration and analysis was not done as this procedure is painful and often associated with conditions that result in a weakened immune system or low platelet count. A weakened immune system can make the patients more prone to infection, and a low platelet count increases the risk of excessive bleeding $[19,20]$.

In most of the studies, hESCs have been derived from 8-cell stage fertilized ovum not used clinically [21]. However, hESCs used in present study were obtained from 2-cell staged fertilized spare ovum obtained after a regular IVF procedure. The hESCS derived at this stage are free from immunogenicity [11]. In our previous studies, we have reported the safety and efficacy of hESC therapy in treating various neurological and non-neurological conditions $[15,22,23]$.

Studies till now focused on transplantation of embryonic stem cell (ESC) derived primary megakar- yocytes, which helps in production of platelets [24, 25]. Human mesenchymal stem cells (hMSCs) produce a variety of hematopoietic cytokines, growth factors, chemokines, such as CXCL12 (SDF-1), Flt-3 ligand (FL), thrombopoietin (TPO), interleukin (IL)-6, IL- 11, leukemia inhibitory factor (LIF), macrophage colony stimulating factor (M-CSF), tumor necrosis factor- (TNF-) $\alpha$, and transforming growth factor(TGF-) $\beta 1$, and also express messenger RNA for TPO, a primary regulator for megakaryocytopoiesis and thrombocytopoiesis [26, 27]. These results suggest that MSCs residing within the megakaryocytic microenvironment in bone marrow provide key signals to stimulate megakaryocyte and platelet production [28]. hESCs used in present study might have shown their therapeutic effect by following the same mechanism.

\section{Conclusion}

The use of hESC therapy in the present study was found to be safe and effective. The patients showed improvement in platelet number. Both patients have not experienced serious AEs during the study as a result of hESC therapy. hESC therapy was well tolerated among both the patients included in the study. No teratoma formation was seen following the treatment. However, clinical studies with large population sizes are needed to gather evidence favoring the use of hESCs in the treatment of thrombocytopenia.

\section{Acknowledgement}

The author acknowledges all the doctors, staff and patients of the Nutech Mediworld. The author also acknowledges Knowledge Isotopes Pvt. Ltd. (http:// www.knowledgeisotopes.com) for the medical writing assistance.

\section{Funding}

No funding sources were used to sponsor this study. 


\section{Competing Interest}

The author declares no competing interest associated with the publication of this manuscript.

\section{Abbreviations}

AEs: adverse events; CAT: congenital amegakaryocytic thrombocytopenia; CP: cerebral palsy; ESC: embryonic stem cell; FL: Flt-3 ligand; GLP: Good Laboratory Practice; GMP: Good Manufacturing Practice; GTP: Good Tissue Practice; hESC: human embryonic stem cell; hMSC: human mesenchymal stem cell; ITP: idiopathic thrombocytopenia; IVF: in vitro fertilization; IL: interleukin; IEC: Institutional Ethics Committee; i.v: intravenous; LIF: leukemia inhibitory factor; M-CSF: macrophage colony stimulating factor; SCl: spinal cord injury; TPO: thrombopoietin; TNF: tumor necrosis factor

\section{References}

1. Izak M, Bussel JB. Management of thrombocytopenia. F1000Prime Rep. 2014; 6:45.

2. Chaudhary B, Jyothi $Y$, Rabbani SI. Thrombocytopenia and its causes. J Chem Pharm Res. 2016; 8(2):184-9.

3. Hassan BAR. Role of cancer and chemotherapy in the incidence of thrombocytopenia. Pharmaceut Anal Acta. 2013; 4:e157.

4. Komur M, Bayram I, Erbey F, Kucukosmanoglu O. A rare cause of thrombocytopenia in infants: vitamin B12 deficiency. Eur J Gen Med 2010; 7(1):107-10.

5. National Heart, Lung, and Blood Institute [Internet]. How is thrombocytopenia treated? [cited 2016 May 15]. Available from: http://www.nhlbi.nih.gov/health/health-topics/topics/thcp/ treatment

6. Royer DJ, George JN, Terrell DR. Thrombocytopenia as an adverse effect of complementary and alternative medicines, herbal remedies, nutritional supplements, foods, and beverages. Eur J Haematol. 2010; 84(5):421-9.

7. Guidry JA, George JN, Vesely SK, Kennison SM, Terrell DR. Corticosteroid side-effects and risk for bleeding in immune thrombocytopenic purpura: patient and hematologist perspectives. Eur J Haematol. 2009; 83(3):175-82.

8. Demetri GD. Targeted approaches for the treatment of thrombocytopenia. Oncologist. 2001; 6(Suppl 5):15-23

9. Lim SH, Kell J, al-Sabah A, Bashi W, Bailey-Wood R. Peripheral blood stem-cell transplantation for refractory autoimmune thrombocytopenic purpura. Lancet. 1997; 349(9050):475
10. Passweg JR, Rabusin M, Musso M, Beguin $Y$, Cesaro S, Ehninger $G$, et al. Haematopoetic stem cell transplantation for refractory autoimmune cytopenia. Br J Haematol. 2004; 125(6):749-55

11. Shroff G. Establishment and characterization of a neuronal cell line derived from a 2-cell stage human embryo: clinically tested cell-based therapy for neurological disorders. Int J Recent Sci Res. 2015; 6(4):3730-8

12. Shroff G, Barthakur JK. Safety of human embryonic stem cells in patients with terminal/incurable conditions- a retrospective analysis. Ann Neurosci. 2015; 22(3):132-8.

13. lajimi AA, Hagh MF, Saki N, Mortaz E, Soleimani M, Rahim F. Feasibility of cell therapy in multiple sclerosis: a systematic review of 83 studies. Int J Hematol Oncol Stem Cell Res. 2013; 7(1):15-33.

14. National Institutes of Health [Internet]. Stem cell information [cited 2015 December 14]. Available from: http://stemcells.nih. gov/info/basics/pages/basics1.aspx

15. Shroff G, Gupta A, Barthakur JK. Therapeutic potential of human embryonic stem cell transplantation in patients with cerebral palsy. J Transl Med. 2014; 12:318.

16. Baraniak PR, McDevitt TC. Stem cell paracrine actions and tissue regeneration. Regen Med. 2010; 5(1):121-43.

17. Al-Ahmari A, Ayas M, Al-Jefri A, Al-Mahr M, Rifai S, El-Solh H. Allogeneic stem cell transplantation for patients with congenital amegakaryocytic thrombocytopenia (CAT). Bone Marrow Transplant. 2004; 33(8):829-31.

18. Takayama N, Nishikii $H$, Usui J, Tsukui $H$, Sawaguchi A, Hiroyama $T$, et al. Generation of functional platelets from human embryonic stem cells in vitro via ES-sacs, VEGF-promoted structures that concentrate hematopoietic progenitors. Blood. 2008; 111(11):5298-306.

19. Healthline [Internet]. Bone marrow aspiration [cited 2016 August 10]. Available from: http://www.healthline.com/health/ bone-marrow-aspiration\#Overview1.

20. Toi PC, Varghese RG, Rai R. Comparative evaluation of simultaneous bone marrow aspiration and bone marrow biopsy: an institutional experience. Indian J Hematol Blood Transfus. 2010; 26(2):41-4.

21. Chung $Y$, Klimanskaya I, Becker S, Li T, Maserati M, Lu SJ, et al. Human embryonic stem cell lines generated without embryo destruction. Cell Stem Cell. 2008; 2(2):113-7.

22. Shroff G, Gupta R. Human embryonic stem cells in the treatment of patients with spinal cord injury. Ann Neurosci. 2015; 22(4):208-16. 
23. Shroff G, Das L. Human embryonic stem cell therapy in cerebral palsy children with cortical visual impairment: a case series of 40 patients. J Cell Sci Ther. 2014; 5:189.

24. Shiraga M, Ritchie A, Aidoudi S, Baron V, Wilcox D, White G, et al. Primary megakaryocytes reveal a role for transcription factor NF-E2 in integrin alpha aiibß3 signaling. J Cell Biol. 1999; 147(7):1419-30

25. Eto K, Murphy R, Kerrigan SW, Bertoni A, Stuhlmann H, Nakano $\mathrm{T}$, et al. Megakaryocytes derived from embryonic stem cells implicate CalDAG-GEFI in integrin signaling. Proc Natl Acad Sci U S A. 2002; 99(20):12819-24.

26. Li T, Wu Y. Paracrine molecules of mesenchymal stem cells for hematopoietic stem cell niche. Bone Marrow Res. 2011; 2011:353878.

27. Mishima S, Nagai A, Abdullah S, Matsuda C, Taketani T, Kumakura $S$, et al. Effective ex vivo expansion of hematopoietic stem cells using osteoblast-differentiated mesenchymal stem cells is CXCL12 dependent. Eur J Haematol. 2010; 84(6):538-46.

28. Cheng L, Qasba P, Vanguri P, Thiede MA. Human mesenchymal stem cells support megakaryocyte and pro-platelet formation from CD34(+) hematopoietic progenitor cells. J Cell Physiol. 2000; 184(1):58-69.

Publish in International Archives of Medicine

International Archives of Medicine is an open access journal publishing articles encompassing all aspects of medical science and clinical practice. IAM is considered a megajournal with independent sections on all areas of medicine. IAM is a really international journal with authors and board members from all around the world. The journal is widely indexed and classified Q2 in category Medicine. 